\title{
Business Acumen in Nursing Management
}

\author{
Katherine Chubbs, RN, BN, MHS, CHE \\ President, Academy of Canadian Executive Nurses \\ Vice President and Chief Nursing Officer, \\ Eastern Health, St. Johns, NL \\ Chair of Canadian College of Health Leaders, \\ Newfoundland Chapter
}

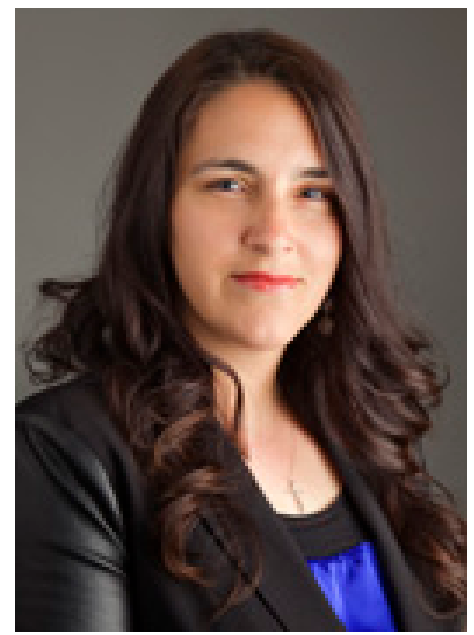

We all know many great front-line nurses who have gone on in their career to become managers. Good performance, as it should, opens the doors of opportunity. Ideally, as nurse managers develop the ability to perform nursing work in a proficient manner, they are able to identify areas for growth in nurses whom they lead and also can act as a role model for nurses reporting to them.

A front-line nurse manager should be many things. He or she should have comprehensive knowledge as well as competency in skills, compassion, empathy and respect - but the list does not end there. Today's nurse manager must also be business oriented.

There is a plethora of articles available on the qualities of a great nurse manager. What's not well documented is the growing demand for nurse managers to have a sense of the business of healthcare. As a service setting, healthcare is constantly challenged to do more with less, to increase output with decreased financial input and to deliver better-quality care to increasing numbers of people. Nursing managers, a large proportion of the healthcare organization's management team, must be equipped to face these changing and growing demands and to develop the knowledge necessary to ensure that the services and team they oversee meet the established goals.

Nurse managers cannot leave the worrying about their organization's financial situation to the finance department. Responsibility for managing the finite resources of healthcare has become a large component of the nurse manager's role. The perspective that we will spend whatever it takes to get the work done is no longer acceptable. Nurse managers are more frequently becoming tasked with 
setting financial goals for their departments. This could take the form of reducing spending on a specific component of their service, such as supply costs. Nurse managers are expected to have intimate knowledge of their budgets and spending and to be accountable for variances in these.

The evolving requirement that nursing managers bear more direct accountability for organizational costs is a positive one. Increased accountability usually means increased attention and, often, increased efficiency. More knowledge of the business side of healthcare strengthens nurses' ability to lead healthcare organizations in a more comprehensive and effective way.

Some may question why we need to consider healthcare as a business. The majority of healthcare services are not-for-profit in our country and, in many cases, our funding is not based on a reward system for performance. The fact remains, though, that we do have to perform better if we want to continue to deliver highquality healthcare. We must look at the foundational elements of the business world to help guide us in achieving this objective.

Two terms are used to understand business practices: effectiveness and efficiency. Effectiveness means making the right decisions about what products or services to offer clients and the best ways to produce or procure them. Efficiency refers to producing products or services quickly while maintaining quality and minimizing waste (Burrow et al. 2008). Some organizations are very effective but are consistently over budget; others are very efficient but their products or services fail to meet quality standards. Nurse managers must focus on both efficiency and effectiveness. Healthcare must have a high level of quality but also be sustainable.

Effectiveness is achieved through having a solid understanding of what those whom you serve need. As a nurse manager, your staff also falls into the category of those for whom you provide. As with any leadership role, nurse managers must be able to influence others. This could mean motivating your team to embrace a change, or stimulating the organization to move in a new direction. Nurse managers need to be aware of the larger environment in which they work. This enables them to identify opportunities for growth while understanding any risks or barriers a change could present. Nurse managers must also have the ability to engage others. Engagement with staff, colleagues and patients is key to successfully managing a program within a healthcare setting. Nurse managers must be able to communicate effectively to clients, staff and other stakeholders. They must be able to mentor front-line nurses. Finally, nurse managers must understand the evidence pertaining to their clinical area and be able to apply that knowledge to ensuring that their service delivers what is required. 
The nurse manager's ability to achieve effectiveness can be influenced by the availability of resources. There is a lot of competition for public dollars, and sometimes no option for an increased budget. Nurse managers must often rely on their ability to achieve internal efficiency to enhance effectiveness.

Efficiency is measured by output and reflects the quantity of something produced over a defined period of time, whereas productivity is defined as producing the greatest quantity in the least amount of time using efficient methods and modern equipment (Burrow et al. 2008). Nurse managers are tasked with ensuring the productivity of their team members. They must be very familiar with individual performance management. Though notoriously irregular when it comes to completing performance reviews, healthcare managers must become attuned to the need to manage performance with a goal to achieving outcomes. Healthcare strategies are usually designed with an evaluation component that describes metrics to be monitored. These metrics are the gauges that highlight success or failure when it comes to meeting the goals set forth in the strategy. Nurse managers are held accountable for ensuring their teams perform in a manner that meets the targets established. Workers are more productive when they are well trained and well managed (Burrow et al. 2008).

The healthcare system has an abundance of data. Nurses collect workload data, and there is financial data, outcome data and so on. Many nurse managers say they do not have time to gather data, look at the data once compiled or know how to use it to influence decision-making. We must create an environment where time can be devoted to data collection and analysis. Funding decisions are often based on evidence and data; therefore, nurse managers must be data driven.

Not all healthcare decisions can be driven by a solid business case. Not every new technology or service can save money, or at least remain cost neutral, while still increasing quality. However, many can. Nurse managers must be aware of the need for a strong business case and understand that such a foundation garners support and stimulates innovation.

The challenge becomes how nurse managers are prepared for this evolution of a business focus in nursing management. Basic educational preparation for nurses touches only minimally on the business side of healthcare. Despite the fact that the decision-making of front-line nurses greatly influences the ultimate cost of healthcare, little education is delivered in basic nursing programs on how to be a steward of limited healthcare dollars. Though this is an ideal focus for future education development, we have to focus now on how to develop that business sense in nurse managers. 
Many healthcare organizations are striving to strengthen internal capacity to develop business acumen in nurse managers. There is a pressing need to build awareness, among all nursing professionals, of the limitations in healthcare resources. Nurse managers should be encouraged to develop their skills from a business perspective. Everyone in healthcare has a responsibility for ensuring the system is sustainable. Managing the largest healthcare workforce, nursing managers must take a leading role.

\section{References}

Burrow, J.L., B. Kleindl and K.E. Everard. 2008. Business Principles and Management. Mason, OH: Thomson South-Western.

\section{Breakfast with the Chiefs is not for everyone.}

\section{You must have an appetite for good ideas!}

